\title{
Functions of an external medical advisory committee
}

\author{
M HARBOE \\ University of Oslo, Institute of Immunology and Rheumatology, \\ Fr. Qvamsgt. 1, N-0178 OSLO 1, NORWAY
}

In considering the functions of the Medical Advisory Committee (MAC) of ALERT, this paper will describe general matters of essential importance for the function of external medical advisory committees in general, and the main topics dealt with at ALERT.

\section{Basis of the work}

In considering the basis of our work, two factors spring to mind immediately.

Firstly, a proper structure is essential.

ALERT has had a Medical Advisory Committee since 1966. However, a profound change was introduced on the initiative of the Executive Director, Bernt Johannessen, in 1980, based on the following principles.

$i$ The committee should consist of only a few members, selected to cover various areas of leprosy.

ii The committee should meet on the local site, in Addis Ababa.

iii The committee should have a clear mandate.

iv The meetings were to be well prepared in advance by the ALERT Management, and specific questions and topics presented.

$v$ The committee were to report directly to the Board, immediately after its meeting which was usually held once a year.

The previous composition of ALERT's Medical Advisory Committee was certainly more distinguished as regards leprosy, but we were now given better opportunities, and the committee became more functional.

The general principle and lesson to be learnt are clearly that: Proper structures are required to provide a sound basis for the work of external medical advisory committees of this kind. 
Secondly, the question of confidence is essential. To improve conditions, difficulties and inadequacies must be made evident, questions must be raised, and criticism becomes a frequent ingredient in the work. It is easy to criticize, but it is far more difficult, but nevertheless essential, to do it in such a way that it becomes a basis for improvement. The committee attempted to do this, and was met with real confidence and open minds at ALERT. "Locally, within the institution, confidence was established, and conditions for work were thus very good. Open criticism such as this, as a basis for improvement of conditions, carries a greater risk of being misunderstood by the institution's external contacts. This risk must be anticipated and accepted by committees of this kind and the sponsors.

\section{Main matters for consideration and development}

A. Multiple Drug Therapy (MDT)

The introduction of MDT on a large scale requires extensive resources, development of infrastructures, and detailed instructions concerning procedures. It became evident that the resources available would not be sufficient to allow full coverage of the areas with MDT. Thus, the first question was how to select parts of the areas covered by the ALERT rural leprosy control program and allocate priorities to these as regards the introduction of MDT.

It was decided that the MDT program should be introduced stepwise, area by area. This principle made it possible to introduce cohort analysis in detailed reporting. Furthermore, the importance of learning from experience was stressed from the start so that experience from one area could later be directly built on in the next. MDT was introduced and established according to the regimens recommended by wHO (1).

It became evident, however, that more detailed instructions were required for local Ethiopian conditions. A manual was therefore prepared by ALERT in collaboration with the National Leprosy Control Program of Ethiopia (2) based on the initial assignment of Dr. A. de Rijk as a WHO temporary advisor to the National Leprosy Control Program and subsequent intensive discussions between the Medical Advisory Committee and senior staff members at ALERT and the National Leprosy Control Program. 
Planning and development of operations then proceeded according to the instructions in the manual. Experience showed that planning in a given area is essential before MDT is introduced, involving a complete coverage of the leprosy population with reclassification of cases into multibacillary (MB) and paucibacillary (PB) cases. The cohort analysis showed very distinct patterns in the various areas, as described by Dr. Becx later in this volume.

The great challenge now and in the next few years is how to extend the analyses to form a penetrating analysis of the effect of the MDT, administered according to the recommended WHO regimens on the leprosy program itself, and particularly on the leprosy endemic in rural and urban areas.

\section{B. The hospital}

The committee has repeatedly evaluated the quality of work at ALERT in view of the responsibilities of the institution as an international training centre for leprosy workers. This requires that senior staff members have extensive knowledge of leprosy and that diagnostic procedures and clinical work are of a consistently high standard.

When acquiring new clinical staff members, the need for a senior leprologist with a profound, detailed knowledge of the disease has been pointed out several times by the committee. This is, of course, a sensitive matter as regards the staff. However, it has far wider implications than those affecting the the ALERT staff.

We live in a phase of transition, and this question is a major challenge to the leprosy field. When the people of my generation who have worked mostly or exclusively with leprosy are gradually being replaced by younger people working in more integrated systems that care not only for leprosy, but also for patients with other, related diseases, a key point becomes evident:

How can we ensure that they will have adequate, in-depth knowledge and clinical experience of leprosy? This is a burning question, and it is essential, if the quality of leprosy work is to be maintained in the future, that $a$ satisfactory answer be found.

In a training institution, the interface between regular clinical work and clinical research becomes essential. 
ALERT has a clear responsibility in carefully studying its large patient material. In the current context of introducing MDT on a grand scale, the development of methods for differential diagnosis of reversal reactions as distinct from relapse after cessation of therapy becomes essential. The two conditions are conceptually distinct, but our present methods of clinical differential diagnosis are clearly inadequate. The question of histopathological examinations and the histopathology of immunological marker systems in tissue specimens needs to be intensively explored for this purpose. Further, the committee has advised that the development of a "Grand Round" system with systematic consideration of leprosy from various points of view such as clinical features, histopathology, and prophylaxis becomes essential. In these ventures, training aspects should be incorporated into the system and regularly reviewed by clinical staff members.

\section{Training}

Considering the essential training functions at ALERT, the committee has analysed the training programs from the point of view that training should be task oriented, embracing knowledge, skills and attitude, with an emphasis on the importance of the final point.

Participants need to be carefully selected to ensure that previous experience and knowledge correspond to the requirements for the individual courses. Further emphasis needs to be given to the training of trainers to increase the effect of teaching at the institution.

The training of the ALERT staff members themselves, has always been considered to be of major importance for the quality and impact of the training activities. Courses in teaching methodolgy for ALERT staff have been introduced on the initiative of the committee.

The issue of integration has been considered, and the committee has taken part in the discussions on the introduction of training in tuberculosis at ALERT and on its significance, not only for tuberculosis work, but for the integrated activities of leprosy and tuberculosis control programs. 


\section{Research}

The committee considers research to be an essential part of ALERT's function as an international training centre. Research is essential to stimulate the quality of the work, and to keep it up to the standard of an international training institution. The compound including both ALERT and the Armauer Hansen Research Institute (AHRI) is unique in combining these two activity areas. Division of work between ALERT and AHRI is essential to avoid duplication and to exploit the advantages of each institution. Catchwords are collaboration and interdependence as illustrated by Dr. Britton in his paper on this issue.

\section{E. The laboratory.}

The need for laboratory work of high quality is obvious in a training institution like ALERT, both in regular clinical work used as a basis for training, and in leprosy control work in the field based on MDT. Together with staff members, the committee has markedly improved the quality of work. As part of these activities, the principle of quality control was introduced in ALERT, apparently the first institution in Ethiopia to start this on a regular basis. This work has formed the basis for quality control in an additional, international context (3).

The quality control procedures concerning smear taking and readings should cover the complete chain of events, from the taking of smears at a local clinic in the rural areas to the reading of the smear in the laboratory and the returning of results to the peripheral clinic. Further procedures should be considered with regard to the distinction between $B I$ of 1 or 2 which is of major importance for the treatment and further handling of the patients according to the WHO recommended MDT regimens.

\section{General considerations}

An external advisory body, with members who feel associated to particular institution, appears to represent a valuable tool for continual evaluation and for initiating changes and improvements. In this work, care should be taken not to overload the system but to work on long term plans giving priority to one area at a time, so that the institution can 
respond adequately to the external advice.

We expect the principle will also be valuable in other institutions in which proper confidence can be established between local members of staff and the external advisory committee, if they are able to accept the challenge as ALERT has done and permit a body of independent advisors to scrutinize the institution as we have done here.

\section{References}

1. Chemotherapy of leprosy for control programmes. Wld Hlth Org Techn Rep Ser, 1982; No. 675.

2. Manual for the implementation of multiple drug therapy (MDT) in Ethiopia. Leprosy Documentation Service (INFOLEP), Amsterdam, 1983.

3. de Rijk AJ, Nilsson T, Chonde M. Quality control of skin smear services in leprosy programmes: preliminary experience with inter-observer comparison in routine services. Lepr Rev, 1985; 56: 177-191. 